\title{
Autismus-Spektrum-Störungen und ihre Bedeutung in der Psychotherapie Erwachsener
}

Andreas Riedel, Ludger Tebartz van Elst, Jens Jürgen Clausen

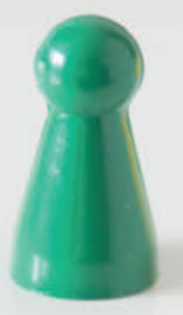

Quelle: Anna-Luise Vogel/Thieme Gruppe

Autismus ist derzeit - medial betrachtet - ein durchaus „modisches“ Phänomen: Dokumentationen, Bücher und Filme zum Thema werden jedes Jahr mehr, und Fernsehserien und Kinofilme „schmücken“ sich immer häufiger mit „Quotenautisten“. Insofern kann durchaus der Eindruck aufkommen, dass es sich bei Autismus um eine Modediagnose handelt, die ebenso schnell wieder verschwinden wird wie sie aufkam. Für PsychotherapeutInnen liegt damit die Folgerung nicht fern, dass man das Thema getrost ignorieren sollte. Diesen Fehlschluss zu widerlegen, ist zentrales Anliegen dieses Artikels.

Im Kontrast zu der medialen „Autismuswelle“ hat sich in der psychiatrischen und psychotherapeutischen Versorgung erwachsener Autisten über die letzten Jahrzehnte wenig bewegt. Betroffene haben nach wie vor das Problem, dass sich niemand für sie zuständig fühlt. Dabei verursachen Autismus-Spektrum-Störungen (ASS) auch im Erwachsenenalter sehr häufig psychiatrische Komorbiditäten und hohen Leidensdruck. Viele Studien zeigten, dass erwachsene Autisten zwar oft gut ausgebildet, aber vom ersten Arbeitsmarkt ausgeschlossen sind, d. h. arbeitslos, berentet oder dauerhaft arbeitsunfähig [1, 2, 3]. Bis zu $50 \%$ der Betroffenen haben sekundäre depressive Störungen $[2,4]$. Es existieren nur wenige diagnostische Zentren für Erwachsene mit Autismusverdacht und - nach unserer Kenntnis - weniger als 25 stationäre Behandlungsplätze im deutschsprachigen Raum. Wir werten diese Fakten als Hinweise, dass die psychiatrisch-psychotherapeutische Versorgung von Erwachsenen mit ASS in Deutschland massiv verbesserungsbedürftig ist.

\section{Was ist Autismus? - Einordung und Historisches}

Im ICD-10 wird Autismus unter dem Überbegriff „tiefgreifende Entwicklungsstörungen“ zusammengefasst und in 3 Unterkategorien unterteilt:

- frühkindlicher Autismus,

- atypischer Autismus

- und Asperger-Syndrom. 
Das DSM- 5 behandelt Autismus unter dem Überbegriff der „neuronalen und mentalen Entwicklungsstörungen“, zu denen u. a. die ADHS, die Tic-Störungen, Intelligenzminderungen und die Lese-Rechtschreibstörung gehören, und arbeitet mit dem Regenschirmbegriff „Autismus-Spektrum-Störung“ (ASS). Dieser fasst alle bisherigen Unterkategorien von Autismus als Dimensionen ein und desselben Phänomens zusammen. Die ICD-11 wird dem DSM-5 in weiten Teilen folgen.

Historisch betrachtet entstammt der Begriff „Autismus“ der Psychoseforschung (Eugen Bleuler [5]). Im Zusammenhang mit Krankheitsbildern, die wir heute als ASS beschreiben würden, wurde er erstmals von den österreichischstämmigen Ärzten Leo Kanner und Hans Asperger aufgegriffen: Kanner beschrieb 1943 unter dem Titel Autistic disturbances of affective contact 11 Kinder mit schweren Auffälligkeiten in der zwischenmenschlichen Kontaktaufnahme und Kommunikation, bis hin zur „Eingekapseltheit" von Geburt an [6]. Asperger berichtete 1944 unter dem Titel Die „Autistischen Psychopathen“ im Kindesalter von 4 Fällen männlicher Heranwachsender, die ebenfalls Schwierigkeiten in der sozialen Interaktion und Kommunikation zeigten [7]. Die von Kanner beschriebene Entität („frühkindlicher Autismus“) wurde bereits in den 1950er Jahren wissenschaftlich diskutiert und klinisch diagnostiziert; das „Asperger-Syndrom“ dagegen fand erst nach einer Übersetzung ins Englische in den 1980er-Jahren Eingang in die üblichen Diagnosesysteme.

\section{Was ist Autismus? - Symptomatik und Erklärungsansätze}

Im Zentrum der Symptomatik stehen nach ICD-10

- die qualitative Beeinträchtigung der wechselseitigen sozialen Interaktion,

- die qualitative Beeinträchtigung der Kommunikation

- sowie repetitive und stereotype Verhaltensmuster und ausgeprägte Sonderinteressen.

Im DSM-5 beinhalten die Diagnosekriterien zusätzlich die Domäne der „sensorischen Besonderheiten“. Konkrete Schwierigkeiten, die sich hinter diesen Begriffen verbergen können, sind in Infobox 3 zusammengefasst.

\section{Hypothese der verminderten Mentalisierungsfähigkeit}

Die wahrscheinlich bekannteste kognitionspsychologische Hypothese zu Autismus ist die der fehlenden oder verminderten „Theory of Mind“ oder „Mentalisierungsfähigkeit“. Damit ist die Fähigkeit gemeint, sich ein Bild vom inneren Zustand des jeweiligen Gegenübers zu machen. „Innerer Zustand“ umfasst die Gefühle der Person, ihre Bedürfnisse, Intentionen, Gedanken und ihr Vorwissen. Erstaunlicherweise haben neurotypische Kinder schon sehr früh ein inneres Modell davon, wer was schon weiß - etwa, wem welche dritte Person schon bekannt ist und wem sie er- klären müssen, wer das ist [8]. Bei Menschen mit ASS entsteht dieses Modell sehr viel später [9]. Häufig können sie auch nur reduziert mentalisieren, was das Gegenüber interessieren oder auch langweilen könnte. Die Fähigkeit zum synthymen Mitfühlen ist bei vielen Menschen mit ASS jedoch deutlich besser ausgeprägt als die Fähigkeit, innere Zustände sachlich zu erfassen; daher sollte aus unserer Sicht nicht von einem „Empathiedefizit“ gesprochen werden. Betont werden muss, dass Erwachsene mit ASS trotz Theory-of-Mind-Defizit durchaus darüber nachdenken können, was im Gegenüber vorgeht. Dieses Mentalisieren passiert jedoch weniger automatisch - sie müssen sich immer wieder daran erinnern, auf den inneren Zustand des Gegenübers zu achten. Interessanterweise springen Mentalisierungsprozesse bei neurotypischen Erwachsenen nicht nur automatisch an, sondern laufen auch wie nebenbei in der Kommunikation mit. Autistische Erwachsene hingegen müssen bewusst und seriell mentalisieren und können sich daher nicht gleichzeitig auf den Gesprächsinhalt konzentrieren.

\section{FALLBEISPIEL}

Ein 52-jähriger promovierter Chemiker berichtet, dass er sich zwar durchaus in andere Menschen hineinversetzen könne, dass er dies aber häufig „vergesse“. Einen Kollegen, der ihm einen großen Gefallen getan habe, habe er beispielsweise vor den Kopf gestoßen, weil er sich nicht bedankt habe. Er sei zwar sehr dankbar gewesen, er habe jedoch „vergessen“, dass der Kollege Wert darauf legen könnte, dass er diese Dankbarkeit auch äußere. An solche Dinge müsse er sich bewusst erinnern. Aus demselben Grund stelle er bei eigenen Vorträgen und in Gesprächen einen regelmäßigen Vibrationsalarm auf seinem Mobiltelefon ein, um sich daran zu erinnern zu prüfen, ob die Zuhörer noch interessiert und bei der Sache seien.

\section{Hypothese der schwachen zentralen Kohärenz}

Eine weitere klassische Hypothese zu ASS ist die der „schwachen zentralen Kohärenz“. Sie erklärt nicht nur die sozialen Symptome von ASS, sondern auch die Besonderheiten des Verarbeitungsstils und die oftmals vorhandenen Sonderbegabungen. In der ursprünglichen Version der Theorie wird Autismus als Defizit ganzheitlicher, holistischer oder „gestalthafter“ Wahrnehmung bei gleichzeitig erhöhten Fähigkeiten zu Detailwahrnehmung und Fehlererkennung beschrieben [10]. Klinisch wird die Theorie durch die Beobachtung gestützt, dass Menschen mit ASS oft bessere Korrekturleser sind, sich oft in Details verzetteln und dabei das „große Ganze“ aus den Augen verlieren. Neuere Untersuchungen zeigen jedoch, dass auch die „schwachen zentralen Kohärenz“ kein kategoriales Defizit ist, sondern vielmehr ein präferierter kognitiver Stil. 


\section{Weitere Hypothesen}

Weitere Hypothesen zu Autismus sind die Spiegelneuronenhypothese, die Konnektivitätshypothese (wenig lange Verbindungen im Gehirn, viele kurze Verbindungen), die Exekutivfunktionshypothese, die Theorie des „Enhanced Perceptual Functioning in Autism“, die „Intense World Theory“, die Hypothese der „zu realen Wahrnehmung“ im Rahmen des „predictive coding“, die Hypothese des Integrationsdefizits, die Monotropismushypothese, die Theorie der mangelnden sozialen Motivation und die „Extreme Male Brain“-Hypothese, die Autismus als Ausdruck einer Extremform des männlichen Gehirns infolge hoher vorgeburtlicher Testosteronexposition auffasst.

Die Autoren dieses Beitrags sehen das Merkmal „Autistische Eigenschaften“ als dimensional an, analog zum Merkmal „Körpergröße“, und nicht kategorial wie das Merkmal „Schwangerschaft“ $[11,12]$. Aus der dimensionalen Struktur von Autismus ergibt sich, dass es keine natürliche Grenze zwischen autistischen Zügen als Normvariante und ASS als Diagnose gibt - diese Grenze muss immer mit einem gewissen Maß an Willkür festgelegt werden.

\section{INFOBOX 1}

\section{Fakten zu ASS}

- Prävalenz ca. $1 \%$, weltweit weitgehend ähnlich

- stabile Kernsymptomatik über die ganze Lebensspanne

- Geschlechterverhältnis w: $m=1: 4$

- in $90 \%$ der Fälle: genetische Ursachen

- in $85 \%$ der Fälle: komplexe polygenetische Vererbung unter Beteiligung von über 100 Genen

- selten monogenetische Ursachen (z. B. fragiles X-Chromosom)

- sehr selten organisch-exogene Ursachen (z. B. Valproat in der Schwangerschaft)

- fließende Übergänge zu anderen tiefgreifenden Entwicklungsstörungen (z. B. ADHS, TouretteSyndrom, Intelligenzminderung [DSM-5])

- Autisten sind nicht gefühllos und nicht empathielos; Autismus sollte nicht mit Psychopathie/ Soziopathie verwechselt werden.

- Autismus wird nicht durch „die Kühlschrankmutter" verursacht.

- Autismus wird nicht durch Impfungen verursacht.

\section{Autismus in der Psychotherapie - was es diagnostisch zu beachten gilt}

Hochfunktionale Formen von Autismus, insbesondere das Asperger-Syndrom, wurden erst in den 1980er- und 1990er-Jahren in die diagnostischen Kataloge aufgenommen; bei Betroffenen, die bis in die 1980er-Jahre noch Kinder waren, wurden daher kinder- und jugendpsychiatrisch auch keine adäquaten Diagnosen gestellt. Viele von ihnen tauchen bis heute mit fehlenden oder falschen psychiatrischen Diagnosen, falschen kausalen Zuschreibungen und falschen Behandlungsansätzen in psychotherapeutischen Praxen auf - durch jahrelange Fehlzuschreibungen und Fehlbehandlungen oftmals frustriert vom medizinischen System. Aus dieser „diagnostischen Lücke“ für Menschen mit hochfunktionalem Autismus der Jahrgänge vor 1980 resultiert ein großer Bedarf an „nachzuholenden Diagnosen“. Vermutlich sind in psychotherapeutischen Praxen mehr unerkannte Erwachsene mit ASS anzutreffen als üblicherweise angenommen.

Die Diagnose wird nach NICE-Guidelines und den deutschen S3-Leitlinien zur Autismusdiagnostik im Erwachsenenalter klinisch gestellt. Bestandteile der Diagnostik sind in Infobox 2 zusammengefasst.

\section{INFOBOX 2}

Wesentliche Bestandteile der Diagnostik

- Fragebögen (AQ, EQ, SRS, ASAS u.v. m.)

- Eigenanamnese (auch bzgl. Kompensationsmechanismen)

- Fremdanamnese für die Kindheit

- ergänzter psychiatrischer Befund

- strukturierte Verhaltensbeobachtung (z. B. ADOS)

- Testpsychologie (z. B. MASC, Wisconsin Cardsorting Test, Gnosis facialis)

\section{Eigenanamnese}

Unbedingt beachtet werden muss, dass Symptome, die im Kindesalter noch beobachtbar waren, im Erwachsenenalter durch Kompensationsmechanismen verborgen werden können. Um sozial nicht aufzufallen unterdrücken Erwachsene mit ASS oftmals stereotype Bewegungen, Echolalie, Wortwiederholungen, laute Selbstgespräche und die eigene Routineorientierung, wie das Tragen immer gleicher Kleidung oder das Essen immer gleicher Lebensmittel. Nicht selten üben Jugendliche mit ASS stundenlang vor dem Spiegel Mimik, um weniger desinteressiert zu wirken. Indem sie soziale Regeln bewusst erlernen, z. B. durch das Lesen des „Knigge“, Flirtseminare, soziales Kompetenztraining oder Schauspielunterricht, können Menschen mit ASS ein differenziertes, bewusst gesteuertes Verhaltensrepertoire entwickeln, das nach komplexen Algorithmen abgerufen werden kann. Solche Kompensationsmechanismen sind schwer beobachtbar, aber recht gut erfragbar; die Eigenanamnese sollte bei der ASS-Diagnostik im Erwachsenenalter daher sehr viel höher gewichtet werden als bei Kindern und Jugendlichen. Infobox 3 gibt die „11-Punkte-Liste“ wieder, anhand derer eine orientierende Eigenanamnese bezüglich Autismus - auch in einer psychotherapeutischen Praxis - durchgeführt werden kann. 


\section{INFOBOX 3}

Eigenanamnestische Fragen zur Orientierung im psychotherapeutischen Setting

Antwortmöglichkeiten, die auf ASS hinweisen, sind jeweils kursiv gesetzt.

1. Blicksteuerung und holistisches visuelles Erkennen: Wohin genau geht der Blick bei „Blickkontakt“ - Augen, Mund, Nase, zwischen die Augen? Wird defokussiert, also das Gesicht des Gegenübers nicht scharfgestellt? Prosopagnosie: Wie werden Gesichter wiedererkannt - am Gesamteindruck oder anhand von Einzelmerkmalen wie Narben, Brille, Frisur oder Ohrform? Können Gesichtsausdrücke interpretiert werden, insbesondere auch feinere Ausdrücke wie Langeweile, Interesse, leichte Trauer, unterdrückte Wut, Ekel etc.?

2. Soziale Kommunikation und soziales Verstehen: Wurden die unausgesprochenen Regeln im Kinderspiel, werden die unausgesprochenen Regeln in formalen oder non-formalen Gruppen und in Hierarchien intuitiv verstanden? Oder werden soziale Regeln anhand von Algorithmen (z. B. durch Lesen von Benimmliteratur) kognitiv erlernt? Können Gedanken, Handlungsintentionen, Bedürfnisse und Gefühle der Mitmenschen intuitiv verstanden und repräsentiert werden? Besteht ein Gespür dafür, wo man sich in Gruppen dazustellt, an wen man sich wendet, wann man anfängt zu sprechen? Gibt es einen intuitiven Zugang zu Gruppenprozessen? Werden soziale Situationen und Alltagsgespräche detailliert im Voraus geplant, z. T. mit exaktem Wortlaut und in verschiedenen Varianten?

3. Soziale Einbindung und soziale Bedürfnisse: Besteht im Lebenslängsschnitt ein auffälliges Einzelgängertum? Bestand in Kindergarten und Grundschule kein Interesse am Kontakt mit Gleichaltrigen? Besteht ein Bedürfnis nach zwischenmenschlichen Kontakten (dies kann sehr unterschiedlich ausfallen)? Besteht das Bedürfnis, die überwiegende Zeit des Tages alleine zu sein?

4. Interaktionelle Fantasie: Teilnahme an „So-tunals-ob-Spielen“ als Kind? Verstand er/sie deren Sinn nicht? Fähigkeit, sich Geschichten für Kinder auszudenken, in denen Menschen interagieren, oder besteht eher eine rege Fantasie für Technisches, z. B. für Stellwerke? Fähigkeit, überzeugend manipulativ zu lügen? Oder sind auch kleinere Ausreden und „weiße Lügen“ („Mir gefällt Deine neue Frisur...“) nur nach detaillierter Planung möglich?

5. Sprachpragmatik: Werden Dinge, die indirekt („durch die Blume“) ausgedrückt werden, verstanden? Können Spaß und Ernst intuitiv unterschieden werden? Gelingt die Einbettung sprachlicher Aussagen in ihren jeweiligen Kontext zuverlässig, oder bereitet es oft Mühe zu verstehen, was der
Gesprächspartner meint? Werden Metaphern und Sprichwörter primär konkretistisch verarbeitet und erst in einem zweiten Schritt in ihre übertragene Bedeutung übersetzt oder gar nicht verstanden? Bereitet das Verstehen von Sprechakten Mühe (z. B. bedeutet „Haben Sie eine Uhr an?“ in den meisten Situationen nicht: „Ich möchte wissen, ob Sie eine Uhr tragen“, sondern: „Bitte sagen Sie mir die Uhrzeit“)? 6. Routinen und Rituale: Ausgeprägtes Bedürfnis nach gleichförmigen, vorhersehbaren Abläufen (beim Waschen, Ankleiden, Essen, bei der Wahl der Wege, bei der Gestaltung des Morgens)? Sollten Dinge immer am gleichen Platz stehen? Bestehen Veränderungsempfindlichkeit und Störanfälligkeit von Abläufen? Wären immer gleiche Tage wünschenswert? 7. Motorische und verbale Stereotypien: (Innere) Echolalie, werden bestimmte Phrasen und Gesten stereotyp verwendet? Gibt es häufige laute Selbstgespräche, „Flattern“ mit den Händen, „Schaukeln“ mit dem Oberkörper, Hüpfen bei Aufregung? 8. Sensorische Hochempfindlichkeit, Reizdiskrimination, sensorische Integration: Ungewöhnliche Empfindlichkeit für Geräusche, Berührungen, Licht, Gerüche? Werden z. B. sanfte Berührungen als unangenehm erlebt, stärkere aber als angenehmer? Reduzierte Schmerzwahrnehmung? Wird Sprache vor mittellauten Geräuschkulissen nicht mehr zuverlässig verstanden? Werden leise gleichmäßige Geräusche bewusster wahrgenommen, und sind sie schwer ausblendbar? Häufiges Reizüberflutungserleben, evtl. mit dissoziationsähnlichen Zuständen?

9. Detailwahrnehmung, Priorisierungsfähigkeiten: Werden geringfügige Raumveränderungen, orthografische Fehler, logische Unstimmigkeiten „automatisch“ wahrgenommen? Macht es Probleme, z. B. für ein Referat, Wichtiges von Unwichtigem zu unterscheiden? Tendenz, sich in Details zu verzetteln? Sonderinteressen? Interesse für Muster, Nummernschilder o. ä.? 10. Gedächtnis: Fotografisches, eidetisches oder Tonbandgedächtnis? Werden automatisch beim Erinnern die beteiligten Affekte mitaktiviert? 11. Motorik und Sonstiges: Gab oder gibt es Probleme beim Ballsport? Können Bewegungen eines Mitspielers antizipiert werden? Besteht ein sehr hohes Bedürfnis nach Gerechtigkeit? Ist Langeweile ein unbekanntes Gefühl? Gibt es synästhetische Wahrnehmung, z. B. beim Rechnen oder beim Hören von Musik?

\section{Beobachtung autismusspezifischer Verhaltenseigenheiten}

Wichtig ist es auch, autismusspezifische Verhaltenseigenheiten zu beobachten [13]: Wie ausgeprägt ist die Dialogfähigkeit, v. a. im non-formalen Gesprächsteil? Inwiefern geht der Patient auf den Untersucher adäquat ein und kann 
er eine wechselseitige Form von Smalltalk führen? Fehlt der Einsatz von Blickkontakt, Augenbewegungen, Händedruck, Mimik (z. B. Kontaktlächeln) und Kopfbewegungen (Nicken, Kopfschütteln etc.) oder ist er auffällig? Wird deskriptive und emotionale Gestik eingesetzt? Ist die Gestik mit dem Sprechen synchronisiert? Werden Gesprächslautstärke und Prosodie passend zu Thema und Affekt moduliert? Besteht ein Konkretismus im Sprachverständnis? Wird Ironie spontan verstanden? Werden indirekte Andeutungen verstanden? Haftet der Patient an bestimmten Themen? Wie flexibel kann er sich auf Themenwechsel einlassen? Wird die implizite Hierarchie gewahrt? Werden der Beziehung angemessene Umgangs- und Höflichkeitsformen verwendet? Wie funktioniert die Organisation des Sprecherwechsels, z. B. Fähigkeit, den Untersucher an der richtigen Stelle zu unterbrechen? Besteht ein intuitives Gespür für die gewünschte Antwortlänge? Besteht eine starke Irritierbarkeit durch Nebengeräusche? Werden motorische, mimische oder verbale Stereotypien sichtbar?

\section{Fremdanamnese für die Kindheit}

Ein weiterer zentraler Bestandteil der Autismusdiagnostik im Erwachsenenalter ist die Fremdanamnese für die Kindheit. Sie kann mithilfe des Diagnostischen Interviews für Autismus - Revidiert (ADI-R) oder in offener Form mit den Eltern durchgeführt werden. Schulzeugnisse und Fotoalben sind eine sinnvolle Ergänzung. Wenn möglich, sollte nachgewiesen werden, dass bereits im Kindergarten- und Grundschulalter autistische Symptome vorlagen (C-Kriterium nach DSM-5).

\section{Autismus in der Psychotherapie - was es therapeutisch zu beachten gilt}

Die Kernsymptome von Autismus können weder psychotherapeutisch noch psychopharmakologisch behandelt werden. Deshalb zielt die Psychotherapie nicht darauf ab, autistische Symptome zu „bessern“, sondern es geht darum,

- einen guten Umgang mit den Symptomen zu finden,

- die autistischen Eigenschaften gut in die Identität zu integrieren

- $\quad$ und die Symptome, die nicht zu verändern sind, akzeptieren zu lernen.

Für viele Patienten ist es sinnvoll, in der Psychotherapie der Frage nachzugehen, wie sie ihre Umwelt so gestalten können, dass sie sich darin wohlfühlen. Ebenfalls wichtig ist Psychoedukation: Es sollte erklärt werden, was Autismus ausmacht, wie neurotypische Kommunikation funktioniert und was die vielen Informationen „zwischen den Zeilen“ bedeuten. Die Patienten sollen dadurch nicht angeleitet werden, neurotypisches Sozialverhalten nachzuahmen; vielmehr soll ein Bewusstsein entstehen, in welchen Bereichen der individuelle Patient soziale Kommunikation „verpasst“, nicht versteht oder nicht mit dem „neurotypisch erwarteten“ Ausdruck reagieren kann.
Bei der Behandlung psychiatrischer Komorbiditäten von ASS muss die Therapie oftmals angepasst werden. Ausführlich beschrieben sind diese Modifikationen in [14]. Eine manualisiertes Herangehensweise an die Therapie von Erwachsenen mit ASS bietet das FASTER-Manual [15]. Viele der vorgeschlagenen Therapieeinheiten lassen sich auch im Einzelsetting umsetzen, auch wenn sie ursprünglich für Gruppensettings gedacht waren.

\section{Die Art des Fragens}

Menschen aus dem Autismusspektrum werden durch offene Fragen leicht verunsichert und verstehen nicht, worauf ihr Gegenüber hinauswill. Daher ist es sinnvoll, Gespräche mit geschlossenen Fragen zu beginnen. Missverständnisse zwischen autistischen Patienten und neurotypischen Therapeuten sind extrem häufig. In so einem Fall sollte von beiden Seiten so lange sachlich nachgefragt werden, bis sich der Sachverhalt klärt. Therapeuten, die die Arbeit mit Menschen aus dem Autismusspektrum nicht gewohnt sind, mag das zunächst wie endlose Intellektualisierung erscheinen - es hilft den Betroffenen aber oft, sich Worte zu erarbeiten, mit denen sie nach und nach besser verstanden werden.

\section{Therapeutische Haltung}

Als Therapeut ist es in der Therapie von Patienten mit ASS wichtig, zuerst davon auszugehen, dass man nicht weiß, was der Patient will, denkt und fühlt. Vorschnelle Projektionen, Deutungen und Zuschreibungen bringen den Patienten unter Druck und liegen auch häufig falsch. Der Therapeut muss also lernen, seine eigene Theory of Mind kritisch zu hinterfragen und nicht auf seiner therapeutischen Deutungshoheit zu bestehen. Bemerkt der Therapeut Phänomene, die er dem Patienten deutlich machen will, sollte er diese eher in Du-Form als in Ich-Form formulieren und nicht in Andeutungen, sondern klar und deutlich (z. B.: „Wenn ich am Ende der Sitzung aufstehe, bleiben Sie häufig sitzen. Das Signal, dass ich aufstehe, bedeutet am Ende der Sitzung, dass Sie auch aufstehen, sich verabschieden und dann gehen sollen.“).

\section{Expositionstherapie}

Extrem wichtig ist es, zwischen den Phänomenen Angst und Zwang einerseits und Reizüberflutung und Stereotypien andererseits zu differenzieren: Bei Angst-Zwangsdynamiken im Rahmen eines Waschzwanges oder bei einer sozialen Phobie kann die wiederholte Exposition der aversiven Situation (etwa durch den Besuch belebter Plätze oder „schmutziger“ Umgebungen) mit Reaktionsverhinderung sehr hilfreich sein. Auch depressive Patientinnen und Patienten werden oft dazu angehalten, sich gegen einen inneren Widerstand in die Gesellschaft anderer Menschen zu begeben.

So sinnvoll Expositionen in vielen (neurotypischen) Fällen sind, bei Patienten mit ASS stößt das Vorgehen mittels Expositionen deutlich an Grenzen: Die Ursache, weshalb 
Menschen aus dem Autismusspektrum belebte Orte als unangenehm empfinden und sie folglich meiden, ist meist Reizüberflutung. Oftmals können die Betroffenen diese dabei nicht benennen und übernehmen die Deutung der Umwelt, dass sie „Angst“ hätten - die Fehldiagnose „Agoraphobie“ liegt nahe. Wird nun die bei Agoraphobie übliche Expositionstherapie angewandt, so führt dies nicht dazu, dass das unangenehme Gefühl nachlässt, sondern es nimmt durch die verstärkte Reizüberflutung sogar zu. In der Folge kann sich sogar eine sekundäre Angst entwickeln: die ganz realistische Angst vor Reizüberflutung.

Menschen aus dem Autismusspektrum können sich also nicht an Reizüberflutung gewöhnen, etwa durch wiederholtes Aufsuchen von Marktplätzen. Ebenso wenig verbessert sich das Unwohlsein in sozialen Situationen, welches viele Menschen mit autistischen Zügen empfinden, keineswegs, indem sie häufig unter Menschen gehen - es bedeutet nur zusätzlichen Stress. Vielmehr ist es für Menschen mit ASS zentral, dass sie soziale Situation analytisch verstehen: Der Therapeut sollte soziale Situationen und ihre Regeln so detailliert wie möglich erklären, um den Patienten zu helfen, sie zu verstehen, und die Teilnahme an sozialen Situationen sollte individuell und niedrig „dosiert“ werden.

\section{FALLBEISPIEL}

Eine 25 Jahre alte Studentin erlebt schon seit Jahren Anspannungszustände in zwischenmenschlichen Situationen, insbesondere in Gruppen. Über die Diagnosen „soziale Phobie“ und „Agoraphobie“ hat sie gelernt, diese Anspannung als „Angst“ zu benennen. Ihr Rückzugsverhalten wurde als Vermeidung und damit als zu behandelndes Symptom identifiziert. Eine Expositionstherapie erbrachte keine erfolgreiche Habituation, in einer anschließenden tiefenpsychologischen Therapie wurden Situationen aus der Grundschulzeit durchgearbeitet, in denen die Patientin zahlreiche „soziale Misserfolge“ erlebte und Mobbingerfahrungen machte. Zwar erlebte die Patientin dadurch subjektiv eine „Erleichterung“, die Anspannungszustände in sozialen Situationen blieben jedoch bestehen. Schließlich wurde die Diagnose einer ASS gestellt. Es wurde klar, dass die Anspannungszustände nicht aus Angst vor Bewertung resultierten, sondern dass Reizüberflutungssituationen im Sinne autistischer „Overloads“ die Ursache waren. Die Vermeidung bzw. Reduzierung reizüberflutender Situationen durch die Patientin war also durchaus sinnvoll und kein Symptomverstärker. Patientin und Therapeutin waren beide sehr erleichtert, dass das mangelnde Ansprechen auf die Therapien kein Therapieversagen war, sondern aus einem fehlgedeuteten Symptom resultierte.

\section{Psychodynamische Behandlungssettings}

Wenn entsprechende Komorbiditäten vorliegen, können tiefenpsychologische Behandlungen bei ASS durchaus sinnvoll sein. Eindrücklich beschrieben ist dies z. B. bei [16]. Jedoch ist insbesondere bei Deutungen Vorsicht geboten: Erwachsene mit ASS, die Erfahrung mit tiefenpsychologischen Behandlungen gemacht haben, berichten häufig, dass ihre Art zu denken, zu sprechen und mit Gefühlen umzugehen im Rahmen dieser Therapie negativ bewertet wurde. Ihnen wurde quasi unterstellt, dass sie ihre Gefühle „abwehren“, indem sie sich der Welt, dem Zwischenmenschlichen und dem Emotionalen auf sehr rationale und analytische Weise nähern. Ihre natürliche Art zu sein und zu denken wurde so als minderwertig und pathologisch etikettiert. An dieser Stelle sei eindringlich dafür geworben, die beschriebenen Deutungen zu unterlassen und den kognitiven Verarbeitungsstil vieler Menschen mit ASS wertungsfrei als menschliche Variante zu akzeptieren. Auch bei Übertragungsdeutungen ist große Vorsicht geboten: Da autistisches Sozialverhalten fast immer fehlinterpretiert wird, liegen auch intuitive Übertragungsdeutungen meistens daneben (vgl. [14]).

\section{Metaphorische Sprache}

Viele zwischenmenschliche und innerseelische Vorgänge lassen sich gut mit Metaphern beschreiben. Im Umgang mit neurotypischen Menschen gelingt es, in Gesprächen über zwischenmenschliche und innerseelische Vorgänge eine gemeinsame Welt von Metaphern zu finden. Menschen mit ASS erleben Metaphern jedoch oftmals als semantisch unscharfen Sprachgebrauch und stören sich daran. So kann es passieren, dass sich der Patient angewöhnt hat, einfach zu schweigen oder gleichgültig zu nicken, wenn er die Bedeutung blumiger Umschreibungen nicht versteht, sodass der Therapeut gar nicht bemerkt, wenn er nicht verstanden wird. Daher ist es sehr sinnvoll, mit dem individuellen autistischen Gesprächspartner zu klären, inwiefern er gängige oder auch neue Metaphern versteht.

\section{Schlusswort}

Autismus ist zu einem Paradigma für die Einsicht geworden, dass Menschen sehr unterschiedlich sein können, mit sehr voneinander abweichenden Stärken-Schwächen-Profilen. Diese Verschiedenheit kann so weit gehen, dass sich Therapeut und Patient im therapeutischen Setting u. U. nicht mehr intuitiv verstehen. Autismus ist auch zum Paradigma für das Unabänderliche in der Therapie geworden, für das, was seltsam erscheint und dennoch weder beurteilt noch psychodynamisch gedeutet noch abtrainiert werden sollte. Beim Autismus wird dies besonders deutlich, doch gilt es natürlich auch für viele andere Eigenschaften und Eigenarten von Menschen. 
FAZIT

Noch immer werden Autismus-Spektrum-Störungen (ASS) im Rahmen psychotherapeutischer Settings häufig übersehen und nur selten angemessen behandelt. Autismus ist dabei als kaum änderbare Grundstruktur von Stärken und Schwächen zu verstehen, die sehr bedeutsam für die psychiatrischpsychotherapeutische Praxis ist - unabhängig davon, ob sie syndromal im Sinne einer psychiatrischen Störungsdiagnose verstanden wird oder subsyndromal im Sinne eines normvarianten Eigenschaftsclusters. Sowohl für das Selbstverständnis vieler Patienten als auch für die Frage nach der „Schuld“ am seelischen Leiden ist es sehr wichtig, autistische Eigenschaften bei psychotherapeutischen Patienten zu erkennen und ggf. zu diagnostizieren. Ohne korrekte Diagnose werden die für ASS typischen psychosozialen und interpersonellen Konfliktkonstellationen oft falsch gedeutet und inadäquat behandelt. Liegen komorbid mit einer ASS Depressionen, Angsterkrankungen, psychotische Dekompensationen oder Stressreaktionen vor, so muss der therapeutische Zugang an die zugrundeliegende ASS angepasst werden. Dies beginnt bei der Art des Fragens, betrifft Expositionstherapien und Übertragungsdeutungen und geht bis hin zum Umgang mit Metaphern, indirekten Botschaften und Ironie.

\section{Interessenkonflikt}

Ludger Tebartz van Elst hält bezahlte Fort- und Weiterbildungsvorträge sowie Workshops zu verschiedenen neuropsychiatrischen und psychotherapeutischen Themen u. a. im Themenfeld Autismus und erhält Tantiemen aus Buchpublikationen.

Jens Jürgen Clausen hält bezahlte Fort- und Weiterbildungsvorträge sowie Workshops zu verschiedenen Themen u.a. im Themenfeld Autismus und erhält Tantiemen aus Buchpublikationen.

Andreas Riedel hält bezahlte Fort- und Weiterbildungsvorträge sowie Workshops zu verschiedenen neuropsychiatrischen und psychotherapeutischen Themen u. a. im Themenfeld Autismus und erhält Tantiemen aus Buchpublikationen.

\section{Autorinnen/Autoren}

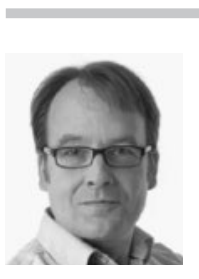

\section{Andreas Riedel}

PD Dr. med. Dr. phil.; seit Mai 2020 Teil eines Leitender-Arzt-Tandems an der Luzerner Psychiatrie im Bereich der ambulanten Dienste; bis April 2020 Oberarzt an der Klinik für Psychiatrie und Psychotherapie der Universitätsklinik Freiburg und stellvertretender Leiter des Erwachsenenbereichs des Universitären Zentrums Autismus-Spektrum (UZAS-Freiburg); seit 2009 Leitung der ambulanten Sprechstunde für hochfunktionalen Autismus im Erwachsenenalter, seit 2016 Leitung der Station für Zwangserkrankungen; Studium der Philosophie und Medizin in Freiburg im Breisgau, Kathmandu und London; aktueller Forschungsschwerpunkt: Sprache bei hochfunktionalen Autismusformen.

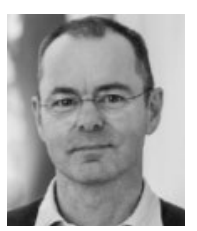

\section{Ludger Tebartz van Elst}

Prof. Dr. med.; Professor für Psychiatrie und Psychotherapie an der Klinik für Psychiatrie und Psychotherapie des Universitätsklinikums Freiburg; Studium der Medizin und Philosophie in Freiburg, Manchester, New York und Zürich; im Rahmen des Post-Docs Forschung am Institute for Neurology/Queen Square, London; stellvertretender ärztlicher Direktor der Klinik für Psychiatrie und Psychotherapie am Universitätsklinikum Freiburg, Leiter der Sektion Neuropsychiatrie und einer der Vorsitzenden des Referats Neuropsychiatrie der Deutschen Gesellschaft für Psychiatrie, Psychotherapie und Nervenheilkunde (DGPPN); seit 2004 Aufbau des Universitären Zentrums Autismus Spektrum in Kooperation mit der Klinik für Psychiatrie und Psychotherapie des Kindes- und Jugendalters in Freiburg; klinische und wissenschaftliche Schwerpunkte: neuronale Entwicklungsstörungen (Autismus, ADHS, Tic-Störungen), schizophreniforme Störungen und organische psychische Störungen.

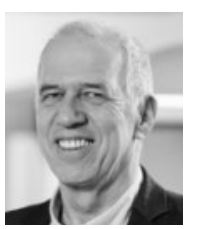

\section{Jens Jürgen Clausen}

Prof. Dr. phil.; Erziehungswissenschaftler und Analytischer Gruppentherapeut; lehrt im Studiengang Heilpädagogik an der Katholischen Hochschule Freiburg; Studium Germanistik, Geschichte und Pädagogik in Hamburg, York (England) und Münster; nach Stationen in Hamm, Münster und Bochum 2012 Berufung auf den Lehrstuhl für Konzepte und Methoden der Heilpädagogik der Katholischen Hochschule Freiburg; Forschungsschwerpunkte: Methodik und Didaktik der Heilpädagogik, historische Grundlagen der Heilpädagogik, der Behindertenhilfe und der Sozialpsychiatrie, Konzepte der Partizipation und Inklusion und institutionelle Entwicklungen in der Eingliederungshilfe, psychische Störungen bei Menschen mit geistiger Behinderung sowie der Themenbereich Partnerschaft, Elternschaft, Sexualität und geistige Behinderung. 


\section{Korrespondenzadresse}

PD Dr. Dr. Andreas Riedel

Luzerner Psychiatrie

Löwengraben 20

$\mathrm{CH}-6004$ Luzern

Schweiz

andreas.riedel@lups.ch

Literatur

[1] Frank F, Jablotschkin M, Arthen T et al. Education and employment status of adults with autism spectrum disorders in Germany - a cross-sectional-survey. BMC Psychiatry 2018; 18: 75

[2] Riedel A, Schröck C, Ebert D et al. Überdurchschnittlich ausgebildete Arbeitslose - Bildung, Beschäftigungsverhältnisse und Komorbiditäten bei Erwachsenen mit hochfunktionalem Autismus in Deutschland. Psychiatr Prax 2015; 43: 38-44

[3] Howlin P. Social disadvantage and exclusion: Adults with autism lag far behind in employment prospects. J Am Acad Child Adolesc Psychiatry 2013; 52: 897

[4] Hofvander B, Delorme R, Chaste P et al. Psychiatric and psychosocial problems in adults with normal-intelligence autism spectrum disorders. BMC Psychiatry 2009; 9: 35

[5] Bleuler E. Dementia praecox oder die Gruppe der Schizophrenien. Leipzig: Deuticke; 1911

[6] Kanner L. Autistic disturbances of affective contact. Nervous Child 1943; 2: 217-250

[7] Asperger H. Die „Autistischen Psychopathen“ im Kindesalter. Arch Psychiatrie Nervenkrankh 1944; 117: 76-136
[8] Perner J. Understanding the representational mind. Cambridge, Mass.: MIT Press; 1991

[9] Baron-Cohen S. Precursor to a theory of mind: Understanding attention in others. Oxford: Blackwell; 1991

[10] Happé F, Frith U. The weak coherence account: Detail-focused cognitive style in autism spectrum disorders. J Autism Development Disord 2006; 36: 5-25

[11] Constantino JN. The quantitative nature of autistic social impairment. Pediatr Res 2011; 69 (5;2): 55R-62R

[12] Tebartz van Elst L. Autismus und ADHS: Zwischen Normvariante, Persönlichkeitsstörung und neuropsychiatrischer Krankheit. Stuttgart: Kohlhammer; 2016

[13] Tebartz van Elst L, Hrsg. Das Asperger-Syndrom im Erwachsenenalter. 2. Aufl. Berlin: MWV; 2016

[14] Riedel A, Clausen J. PraxisWissen: Autismus-SpektrumStörungen bei Erwachsenen. 2. Aufl. Köln: Psychiatrieverlag; 2020

[15] Ebert D, Fangmier T, Lichtblau A et al. Asperger-Autismus und hochfunktionaler Autismus bei Erwachsenen. Das Therapiemanual der Freiburger Autismus-Studiengruppe. Göttingen: Hogrefe; 2013

[16] Saalfrank B. Ich, Birgit, Autistin und Psychotherapeutin. Ostfildern: Patmos; 2019

Bibliografie

DOI https://doi.org/10.1055/a-0987-5653

PiD - Psychotherapie im Dialog 2020; 21: 28-35

(c) Georg Thieme Verlag KG Stuttgart · New York

ISSN 1438-7026 